\title{
LAS DIFERENCIAS NORTE-SUR EN EL DEBATE AMBIENTAL GLOBAL. EL CASO DE LA PROPUESTA DEL ECUADOR: Yasuní - ITT ${ }^{1}$
}

\author{
The North-South differences in the global environmental debate. \\ the case of the Ecuador's initiative: Yasuni-ITT
}

\author{
Fernando Estenssoro Saavedra* \\ Juan Pablo Vásquez Bustamante**
}

\begin{abstract}
RESUMEN
En el presente artículo se plantea que existe una importante contradicción política y de poder Norte-Sur respecto de cómo entender y cómo solucionar la crisis ambiental, que se remonta desde la Conferencia de Naciones Unidas sobre el Medio Humano de 1972. Este debate ambiental global no ha desaparecido de la agenda pública mundial a pesar de los años, sino que fue evolucionando. Esta afirmación se ejemplifica con la propuesta ecuatoriana conocida como Yasuní- ITT, que surgió en los años 90 del siglo pasado, en el marco de la lucha contra el cambio climático.
\end{abstract}

Palabras clave: Yasuní-ITT, Ecuador, crisis ambiental, geopolítica ambiental, ecología política, cambio climático, tensiones Norte-Sur.

${ }^{1}$ Este artículo es producto del proyecto Fondecyt $\mathrm{N}^{\circ} 1150569$, titulado: "Perspectivas Latinoamericanas en el Debate Ambiental Mundial entre 1992 y 2012. Los casos de Chile, Ecuador y Brasil. Un estudio de historia de las ideas políticas del tiempo presente en el espacio de la política mundial e internacional".

* Director del Instituto de Estudios Avanzados de la Universidad de Santiago de Chile. Santiago, Chile. Investigador responsable del proyecto Fondecyt $\mathrm{N}^{\circ} 1150569$. Correo electrónico: fernando. estenssoro@usach.cl

** Doctorando en Estudios Americanos, Universidad de Santiago de Chile. Santiago, Chile. Coinvestigador de proyecto Fondecyt N¹150569. Correo electrónico: juan.vasquez@usach.cl

Artículo recibido el 24 de agosto de 2016. Aceptado el 18 de agosto de 2017. 


\begin{abstract}
This article raises that there is an important North-South political and power contradiction regarding how to understand and how to solve the environmental crisis, that dates back to the United Nations Conference on the Human Environment of 1972. This global environmental debate has not disappeared from the world public agenda despite the years, But has evolved. This statement is exemplified by the Ecuadorian proposal known as Yasuní-ITT, which emerged in the 1990s in the context of the fight against climate change.
\end{abstract}

Keywords: Yasuní-ITT, Ecuador, environmental crisis, environmental policy, political ecology, climate change, North-South tensions.

\title{
INTRODUCCIÓN
}

La idea que enfrentamos una Crisis Ambiental Global está formalmente presente en la discusión política internacional contemporánea desde el año 1972, con motivo de la celebración de la primera Conferencia de Naciones Unidas sobre el Estado del Medio Humano, realizada en Estocolmo en junio de ese año (en adelante Estocolmo 72). En esa oportunidad se emitió la Declaración de Estocolmo que reconocía que la humanidad estaba enfrentando una crisis ambiental global y llamaba a los estados, gobiernos y pueblos del mundo, junto a los organismos internacionales a desarrollar urgentes acciones conjuntas y mancomunadas destinadas a su superación, como bien se desprendía de su punto No 7 , al señalar que había:

un número cada vez mayor de problemas relativos al medio que, por ser de alcance regional o mundial o por repercutir en el ámbito internacional común, requerirán de una amplia colaboración entre las naciones y la adopción de medidas por las organizaciones internacionales en interés de todos. La Conferencia encarece a los gobiernos y a los pueblos a que aúnen sus esfuerzos para preservar y mejorar el medio humano en beneficio del hombre y de su posteridad (Grinberg, 1999, p. 181).

Es a partir de este reconocimiento que podemos sintetizar como debate ambiental global a toda la discusión (acuerdos, protocolos, propuestas y contrapropuestas, otros) que han surgido y surgen en la comunidad internacional destinadas a superar esta crisis. Y, sin lugar a dudas, este debate, amplio y multidisciplinar, se ha constituido en factor crecientemente protagónico de la agenda política mundial, a lo menos desde 1972 a la fecha y todo indica que 
esta situación tenderá a acrecentarse en el presente siglo. En este sentido, desde su nacimiento hasta la fecha, una de las características políticas más importantes de este debate ha sido la tensión Norte-Sur respecto de cómo entender esta crisis ambiental global y, por lo tanto, dónde poner los énfasis para su superación y/o qué respuestas proponer a los problemas planteados (Estenssoro, 2014). En el presente artículo, resultado de una investigación histórica de política internacional focalizada en el acápite ambiental de la agenda mundial, se puede analizar esta situación planteando los aspectos originales de esta tensión y cómo estos vuelven a repetirse, de manera mucho más contemporánea, al analizar el fracaso, en el año 2013, de la propuesta Yasuní-ITT que el gobierno del Ecuador planteó a la comunidad internacional en el marco de discusiones destinadas a superar, en primer lugar, el fenómeno del cambio climático, y en segundo lugar la pérdida de la biodiversidad, que son variables componentes de esta crisis ambiental global.

\section{Antecedentes del surgimiento de la tensión Norte-Sur en el debate ambiental global}

Desde que se instaló formalmente el debate ambiental en la agenda pública mundial, con motivo de la celebración de la primera gran cumbre de la ONU sobre el estado del medio ambiente en Estocolmo 72 se reflejó una clara tensión NorteSur respecto de cómo entender el problema de la crisis ambiental y, por tanto, qué tipo de políticas públicas globales eran necesario enfatizar para su superación. Específicamente, los representantes de los países en vías de desarrollo (entonces identificados como Tercer Mundo) no simpatizaron con la idea de celebrar esta cumbre porque consideraban que era una iniciativa surgida exclusivamente según los intereses de los países primermundistas y/o altamente industrializados. Y, al respecto, estaban en la razón, como bien reconoció el propio Secretario General de esta Conferencia, el canadiense Maurice Strong, al señalar que fue en "los países industrializados donde la preocupación por la contaminación creó la idea original de la Conferencia de Estocolmo" (Strong, 1983: 247). Lo cierto es que en la convocatoria original de la Organización de las Naciones Unidas (ONU) a la celebración de la Conferencia de Estocolmo 72, solo se reflejaban los intereses y perspectivas de los países más desarrollados frente a este problema de la crisis ambiental. El Primer Mundo, menos de un tercio de la humanidad, consciente de que su proceso de desarrollo e industrialización había contaminado su medio ambiente regional y, además, había causado una amenaza global, veían con verdadero pánico la intención del Tercer Mundo -más de dos tercios de la humanidad-, de alcanzar el estándar y estilo de vida del Primer Mundo modernizando e industrializando sus economías.

Advirtiendo, en especial, la deterioración constante y acelerada de la calidad del medio humano causada por los factores tales como la contaminación del aire y de las aguas, la erosión y otras formas de deterioración del suelo, los desechos, 
el ruido, y los efectos secundarios de los biocidas que se ven acentuados por el rápido crecimiento de la población y por la urbanización acelerada [...] Decide [La Asamblea general de la ONU]... convocar en 1972 a una Conferencia de las Naciones Unidas sobre el Medio Humano (A/RES/2398 (XXIII), 1968).

En este sentido, la convocatoria de la ONU no era inocente políticamente. Surgía en medio de un clima intelectual primermundista de claras características neomalthusianas que se venían socializando desde el término de la Segunda Guerra Mundial, tanto en los Estados Unidos como en Europa occidental, por parte de destacados científicos y académicos que, conscientes del daño al ambiente global que el propio Primer Mundo había causado con su proceso de industrialización y desarrollo, consideraban, con extraordinaria preocupación, que los esfuerzos por desarrollarse de los países del Tercer Mundo, así como el aumento creciente de su población, terminaría por agotar los recursos junto con provocar el colapso definitivo del frágil ecosistema planetario. La idea fuerza que manifiestamente querían hacer hegemónica (y lo lograron) era que no había planeta suficiente para que todos sus habitantes tuvieran el estándar de vida del Primer Mundo. Y si bien, en términos generales, con su retórica llamaban a modificar el estilo de vida consumista del Primer Mundo y la creencia en el crecimiento económico permanente, lo cierto es que sus principales esfuerzos los pusieron en destacar los peligros "catastróficos" de la industrialización del Tercer Mundo, así como su "exceso" de población. Esta perspectiva de la crisis ambiental, se comenzó a socializar desde fines de los años cuarenta por una serie de ensayos de divulgación científica y artículos que se convirtieron en verdaderos best sellers y terminaron por hacer hegemónica esta perspectiva en los años de la convocatoria a la Cumbre de Estocolmo. El clímax en la popularidad primermundista de esta argumentación fue el éxito de difusión del primer informe del Club de Roma, Los límites del crecimiento, que sintetizó magistralmente esta particular concepción de la crisis ambiental y que publicado meses antes de la Conferencia de Estocolmo 72, planteó predicciones tales como:

Si no se modifican las tendencias actuales en cuanto a crecimiento de la población mundial, industrialización, contaminación, producción alimentaria y agotamiento de los recursos, alcanzaremos el límite de crecimiento de este planeta en el transcurso de los próximos cien años. El resultado más probable será una repentina e incontrolable caída de la población y la capacidad industrial (Meadows et al., 1972, p. 23).

En otras palabras, la Convocatoria a la Conferencia Sobre el Medio Humano de Estocolmo 72, se dio en medio de un debate caracterizado por "una crítica despiadada del desarrollo (crecimiento) visto por algunos como causa primera del deterioro ambiental" (Bifani, 1999: 105). Por lo tanto, no es de extrañar que este 
discurso provocara una profunda desconfianza entre los representantes del Tercer Mundo. Ellos sospechaban que los países primermundistas estaban orientando los esfuerzos del sistema internacional a priorizar la solución de aquellos temas que venían afectando la calidad de vida de sus ya opulentas sociedades, dejando de lado los esfuerzos por superar el subdesarrollo de la mayoría de la humanidad, como bien reflejó el embajador brasileño en la Conferencia de Estocolmo 1972, al señalar:

En la Conferencia de Estocolmo, los países desarrollados adoptaron una actitud en virtud de la cual se atribuyen, gracias a su desarrollo, un derecho especial a salvarse y perpetuarse, trasladando a los pueblos subdesarrollados, cuya población es más numerosa, la responsabilidad de dejar el espacio necesario en la tierra. Esta actitud es tanto más peligrosa cuanto que tal principio no se discutió públicamente, sino que estaba implícita en los documentos (Ozorio de Almeida, 1973, p. 28).

El malestar de los representantes del Tercer Mundo con esta Conferencia llegó a tal punto que planificaron su boicot, situación que obligó a su Secretario General, Maurice Strong, a realizar acciones urgentes destinadas a diluir estas intenciones (Estenssoro, 2014). La más importante fue cuando Strong llamó a una reunión ad hoc en 1971, en el pueblo Suizo de Founex, a destacadas figuras de organismos internacionales e intelectuales generadores de opinión del Tercer Mundo, a fin de negociar una solución. Después de tres días salió humo blanco, con la denominada Declaración de Founex, en donde se podía leer:

Puede afirmarse que, en gran medida, el actual interés en las cuestiones relacionadas con el medio ambiente ha tenido su origen en los problemas experimentados por los países industrialmente adelantados. Estos problemas son de por sí, en gran parte, el resultado de un nivel elevado de desarrollo económico [...] Estas perturbaciones han llegado a alcanzar tales proporciones que en muchos sitios constituyen ya un grave peligro para la salud y el bienestar humanos [...] Sin embargo, los principales problemas ambientales de los países en desarrollo son básicamente diferentes de los que se perciben en los países industrializados. Son principalmente problemas que tienen su raíz en la pobreza y la propia falta de desarrollo de sus sociedades. En otras palabras, son problemas de pobreza rural y urbana [...] Por estas razones, la preocupación por el medio ambiente no debe debilitar, y no es preciso que lo haga, el compromiso de la comunidad mundial -tanto de los países en desarrollo como de los industrializados- de dedicarse a la tarea principalísima de desarrollar las regiones más atrasadas del mundo. Por el contrario, subraya la necesidad no sólo de comprometerse plenamente a alcanzar las metas y objetivos del segundo decenio para el desarrollo, sino también redefinirlas a fin de atacar la miseria que es el aspecto más importante de los problemas que afligen al medio ambiente de la mayoría de la humanidad ("El Informe de Founex", en Botero y Tokatlian, 1983, pp. 51-85). 
Strong incorporó esta Declaración de Founex a los documentos de convocatoria a Estocolmo 72, asegurando así la participación tercermundista en ella y permitiendo que esta finalizara con éxito, teniendo en la Declaración de Estocolmo y en la creación del Programa de Naciones Unidas para el Medio Ambiente (PNUMA) sus dos mayores resultados.

Sin embargo, a pesar de que la Declaración de Founex permitió instalar la perspectiva del mundo subdesarrollado en el naciente debate ambiental global, al relacionar en una sola ecuación la necesidad de protección del medio ambiente con sus aspiraciones de alcanzar el desarrollo bajo la fórmula Medio Ambiente y Desarrollo, la discusión entre desarrollados y subdesarrollados sobre cómo entender la crisis ambiental y dónde poner los énfasis para su solución, continuaron en los años siguientes. La tensión alcanzó su clímax con el fracaso de la segunda gran Conferencia de la ONU sobre el medio ambiente, pensada para 10 años después de Estocolmo y que se debía realizar en Nairobi en 1982, y en la cual se debía evaluar el estado de la lucha de la humanidad contra la crisis ambiental, así como definir las nuevas políticas a seguir y/o reorientar las existentes.

Lo interesante del fracaso de la Cumbre de Nairobi en 1982 es que llevó a la ONU, en 1983, a formar la Comisión Mundial de Medio Ambiente y Desarrollo (CMMD), a fin de concordar una perspectiva Norte-Sur respecto de la crisis ambiental que permitiera recuperar el llamado de Estocolmo 72 a que toda la comunidad internacional enfrentase unida y mancomunadamente semejante desafío. Encabezada por la noruega Gro Harlem Brundtland, esta comisión, después de cuatro años de arduo trabajo, finalmente evacuó el conocido informe Nuestro Futuro Común (1987), donde se definió en extenso el ya muy conocido concepto de "sustainable development" (desarrollo sostenible o sustentable según se tradujo indistintamente al castellano). De esta forma se unía definitivamente los conceptos de Medio Ambiente y Desarrollo en el debate ambiental mundial y, ahora sí, la ONU pudo retomar con éxito esta idea de realizar cada 10 años una gran Cumbre y/o Conferencia Mundial para evaluar el estado de la lucha de la humanidad contra el deterioro del Medio Ambiente, así como orientar las nuevas tareas al respecto. De esta forma se convocó a la Cumbre de Medio Ambiente y Desarrollo de 1992 en Río de Janeiro (en adelante Río 92) también conocida como Cumbre de la Tierra (Estenssoro, 2014).

Sin lugar a dudas, la cumbre de Río 92 fue la más exitosa de todas a las que se han convocado, desde 1972 hasta el día de hoy, para tratar el tema de la crisis ambiental, precisamente porque ahora sí, bajo el concepto de desarrollo sustentable, estaba mucho mejor recogida la perspectiva de los países en vías de desarrollo. Sin embargo, pese al éxito relativo de Río 92, esto no significó que la inicial tensión Norte-Sur reflejada en Estocolmo 72 desapareciera del todo. Incluso importantes críticas surgieron a los resultados de Río 92 dado que no se avanzó 
de acuerdo a muchas de las expectativas que surgieron en un primer momento, particularmente en los esfuerzo por disminuir la amenaza del Cambio Climático o “calentamiento global". Especialmente se culpó a la actitud economicista asumida por Estados Unidos quien fue ahora el país que recurrió a la amenaza del boicot del evento si no se cambiaba el texto de la Convención sobre el Cambio Climático, "puesto que las transformaciones que estaría forzada a realizar la industria de ese país implicarían importantes pérdidas para la economía”, a lo que se sumaba su insistencia en "solicitar más evidencias científicas sobre las causas del efecto invernadero", lo que llevó a un representante de Greenpeace, a decir que la actitud de los EE.UU. equivalía a "afirmar que se va a contribuir para controlar el incendio, mientras se retiene el derecho de seguir echando bencina" (Guimaraes, 1992, p. 90). Incluso esta postura estadounidense fue apoyada por los países árabes productores de petróleo quienes temían que "la reducción de las emisiones de $\mathrm{CO}^{2}$ provocaría una reducción en el consumo de combustibles fósiles" (Ibíd). Y, como era de esperarse, en los ańos siguientes, esta tensión continuará por nuevos e interesantes derroteros que de una u otra forma demuestran que si bien todos están conscientes de los peligros globales que implica la crisis ambiental, las diferencias de intereses entre desarrollados y subdesarrollados es la más gravitante al momento de alcanzar acuerdos razonables para todos, como se puede ejemplificar con el derrotero de la iniciativa ecuatoriana para mitigar el fenómeno del cambio climático, conocida como Yasuní-ITT.

\section{La iniciativa Yasuni-ITT, en la lucha contra el cambio climático y la pérdida de la biodiversidad}

A fin de terminar con la amenaza de Cambio Climático, en mayo de 1992 Naciones Unidas adoptó La Convención Marco sobre el Cambio Climático (CMNUCC), la que se dio a conocer en la Cumbre de Río 92. En esta Convención Marco los países firmantes (partes) se comprometían, según el principio de responsabilidades comunes pero diferenciadas, a tomar medidas concordadas destinadas a bajar la emisión antropogénica de gases efecto invernadero (GEI) (Naciones Unidas, 1992). Bajo este espíritu se firmó en 1997, el Protocolo de Kioto, que estipulaba medidas vinculantes para las 30 economías más desarrolladas e industrializadas del mundo y voluntarias para las economías en desarrollo, además de proponer mecanismos de reducción de emisiones de GEI como los llamados bonos de carbono. Dado que este acuerdo expiraba en el año 2012, a medida que esta fecha se acercaba las reuniones anuales de la Conferencia de las Partes (COP) se orientaron a avanzar en un nuevo acuerdo que reemplazara al de Kioto y que fuera vinculante para todos las partes. En este espíritu, el año 2007 el Presidente del Ecuador, Rafael Correa, propuso en la Asamblea General de las Naciones 
Unidas, la Iniciativa Yasuní-ITT que, básicamente, consistía en mantener bajo tierra (no explotar) los más de 840 millones de barriles de petróleos encontrados en el subsuelo amazónico del Parque Nacional Yasuní -y que equivalían al 20\% de la producción petrolera del país-, evitando así la generación de 407 millones de toneladas de $\mathrm{CO}^{2}$ a la atmósfera, además de evitar otros efectos nocivos al ecosistema amazónico producto de la actividad extractiva, como la deforestación, la propagación de metano, la migración de especies, contaminación de aguas entre otras consecuencias (Correa, 2007; Gobierno del Ecuador, 2010; Larrea, 2012a; Vásquez, 2014 y 2015).

Para que este compromiso se hiciese efectivo, Ecuador pedía a cambio a la comunidad internacional, una contribución financiera de US\$3.600 millones de dólares según precios internacionales del barril de crudo, equivalente, a lo menos, al 50\% de los ingresos que el país dejaría de percibir por no explotar esta fuente de petróleo. Esta cantidad de dinero debía reunirse en 13 años a partir del 2007, siendo un hito condicionante contar con 100 millones de dólares hacia diciembre de 2011. Estos fondos serían reunidos en un Fideicomiso Internacional (FI), administrado por el Programa de Naciones Unidas para el Desarrollo (PNUD) y gobernado por un "Comité de Dirección", en el cual tendrían participación el Gobierno del Ecuador, los contribuyentes y la sociedad civil ecuatoriana. Para estos propósitos, en agosto de 2010, y tras serias pugnas y crisis en las negociaciones (se analizan más adelante), se firmó un Memorándum de Acuerdo entre el Gobierno ecuatoriano y el PNUD de Naciones Unidas, que creó el Fondo de Fideicomiso Ecuador YasuníITT (Fondo Yasuní). Como elemento garante del cumplimiento del compromiso de mantener el petróleo bajo tierra, el Gobierno ecuatoriano entregaría a cambio a los contribuyentes unos documentos financieros denominados Certificados de Garantía Yasuní (CGY), equivalentes al valor nominal de cada contribución, emitidos a perpetuidad, no intercambiables y que se harían efectivos solamente en caso de que Ecuador incumpliese el acuerdo. Los dineros del Fondo Yasuní se invertirían en el financiamiento de programas estratégicos de desarrollo sustentable definidos en el Plan Nacional de Desarrollo (y que con la Nueva Constitución del Ecuador aprobada en 2008, se transformó en Plan Nacional para el Buen Vivir), todos directamente relacionados a la conservación de la biodiversidad y los ecosistemas, al impulso a las energías renovables, a la investigación, ciencia, innovación y tecnología dirigida al desarrollo de bioconocimiento y al cambio de la matriz energética (Ibíd.).

Por otra parte, como el Ecuador planteaba que el grueso de los fondos solicitados para mantener el petróleo bajo tierra (US \$3.600 millones) debían provenir principalmente de transacciones referenciales ligadas al mercado de carbono, y que las mayores se esperaban de parte de los países industrializados, los cuales se encuentran en el anexo 1 del protocolo de Kyoto, señalaba que su 
"comunidad internacional" interpelada eran precisamente los países del Norte altamente desarrollados e industrializados (Larrea, 2012a, p. 22).

Por cierto, la propuesta del Ecuador señalaba que si no se reunían los dineros provenientes de la comunidad internacional en los plazos establecidos, se llevarían a cabo los planes extractivos en la zona, situación que finalmente fue la que impuso, cuando el 15 de agosto de 2013, el gobierno de Rafael Correa dio por terminada la iniciativa seńalando su fracaso por la falta de una respuesta adecuada de la comunidad internacional, ya que, tras seis ańos de iniciativa solo se habían reunido 13,3 millones de dólares en el Fondo Yasuní, o sea un 0,37\% de lo esperado, además de otros compromisos no directamente vinculados por otros 116 millones de dólares (Correa, 2013).

\section{La propuesta ecuatoriana en la perspectiva histórica del Sur en el debate ambiental}

La iniciativa Yasuní-ITT logró captar la atención y el apoyo de numerosos líderes políticos, personalidades y representantes del ambientalismo y ecologismo nacional e internacional. Se trataba de un paso muy significativo dentro de la perspectiva que el Sur le venía manifestando al Norte, en el debate ambiental, desde Estocolmo 72 en adelante. Desde siempre, para el Sur las fórmulas de superación de la crisis ambiental global, cuyo principal causante era el Norte, debían considerar medidas que facilitaran y no afectaran su interés primordial por alcanzar el anhelado desarrollo. Estos planteamientos, expresados en 1971 en el Informe de Founex, se repitieron y fueron claves para consensuar el concepto de Desarrollo Sustentable que en 1987 propuso la CMMD, e, igualmente, volvieron a tener un avance cualitativo en la Conferencia de Río 92 cuando se señaló que el Norte debía hacerse cargo de la "deuda ecológica" que con su estilo de vida y desarrollo había generado con el Sur.

De esta discusión se avanzó a la aplicación del Principio de Responsabilidades Comunes pero Diferenciadas (PRCD), en temas de cambio climático como un criterio de justicia y equidad ya que de esta manera se permitiría un trato diferenciado entre las partes y por consiguiente una asimetría convencional, dado que era evidente que algunos países - los desarrollados e industrializados- tenían mucho más obligaciones que otros -los en vías de desarrollo- (Arístegui, pp. 585587). Y Ecuador, demostraba tener profundamente internalizada esta perspectiva cuando el presidente Correa señaló, primero en 2008 que "no hay peor enemigo para el medio ambiente que la pobreza” (en El Mercurio, 2008, p. A5), y luego, en 2011 que la "conservación en países pobres no será sostenible si ésta no genera claras y directas mejoras en el nivel de vida de la población” (Correa, 2011b).

Igualmente, con esta iniciativa Yasuní, Ecuador proponía una fórmula concreta para una idea que, por lo menos en América Latina, se venía seńalando desde 
los años 1980, referida a poner valor económico a los múltiples y trascendentales servicios ambientales que prestaban los ecosistema de la región no solo para el mantenimiento del equilibrio del ecosistema planetario, sino que para el propio mantenimiento del alto estándar de vida de las industrializadas sociedades del Norte. Por ejemplo, en 1980 Sunkel y Tomassini plantearon que las sociedades industrializadas del Norte, precisamente por su modo y alto estándar de vida, eran las principales emisoras de dióxido de carbono a la atmosfera. Además, estas sociedades primermundistas se beneficiaban de la capacidad de absorción de este dióxido por parte de los ecosistemas tropicales del Sur y no pagaban un peso por ello. Más aún, dado que la capacidad de los ecosistemas del Norte estaban saturados para realizar estas capacidades de absorción de contaminantes y desechos, ahora comenzaban a trasladar sus industrias a los países del Sur haciendo así, un uso abusivo de este recurso, relativamente escaso, de que disponían los países del Sur: la capacidad de absorción de $\mathrm{CO}^{2}$ de sus ecosistemas boscosos (Sunkel y Tomassini, 1980). Más tarde, Sunkel insistirá en que los ecosistemas son sistemas productivos que proporcionan bienes y servicios y cumplen ciertas funciones ecosistémicas, de gran valor para la sociedad, y en este sentido, la naturaleza se debía entender como un capital natural, puesto que genera un flujo de bienes y servicios indispensables para el desarrollo, por lo tanto los países del Sur debían poner precio a estos servicios y no regalarlos como estaba ocurriendo (Sunkel, 1990).

Lo cierto es que Correa recogió todos estos planteamientos al señalar que los países de América Latina, especialmente los amazónicos, eran doblemente explotados por los países del Norte ya que a la ingente cantidad de recursos financieros que con motivo del pago de la deuda externa transferían al Primer Mundo (y por lo tanto, no podían utilizar en superar sus condiciones de subdesarrollo), se sumaba el hecho de que estos países eran generadores de bienes ambientales fundamentales para el funcionamiento ecosistémico del planeta, tales como el aire puro y oxígeno que generaba la Amazonía, pero estos bienes, al ser considerados bienes de libre acceso, no recibían la "justa compensación por el servicio” que producían (Correa, 2010, p. 204). Por lo tanto, había que avanzar en la creación de una institucionalidad internacional que, junto con valorar estos bienes públicos fundamentales, a su vez pudiera cobrar por el consumo de dichos bienes a los países industrializados contaminadores. Con estos recursos los países del Sur, en primer lugar, se pagarían de la deuda ecológica que el Norte tenía por los daños ambientales ya causados y, en segundo lugar, con dichos fondos el Sur pagaría su deuda externa sin comprometer su desarrollo (Ibíd.).

En síntesis, señalaba Correa, a los países en vías de desarrollo se les debía compensar la contaminación neta evitada, dado que, en este caso de la propuesta Yasuní, al no extraer petróleo para ser usado como combustible junto con evitar la generación de millones de toneladas de $\mathrm{CO}^{2}$ también se protegía la biodiversidad 
y el ecosistema de la región. De esta forma, los "países pobres generadores de bienes ambientales finalmente tendrían la justa compensación por los invalorables servicios" que estaban "suministrando para la vida de todo el planeta, sin necesidad de recurrir a cooperación, canjes, caridades, etcéteras”, además de permitir el "paso de economías extractivistas a economías exportadores de servicios, en este caso, ambientales" (Ibíd, p. 205).

Es desde este punto de vista, que la propuesta que hizo Correa con YasuníITT, recogió y profundizó la perspectiva histórica del Sur en el debate ambiental y se convirtió en un nuevo hito de la demanda de justicia y equidad ambiental que el Sur siempre le ha hecho al Norte al momento de diseñar las políticas tendientes a terminar con la amenaza de la crisis ambiental global.

\section{EL FRACASO DE LA INICIATIVA YASUNÍ-ITT}

Sin embargo esta interesante propuesta fracasó, demostrando que la inicial tensión histórica Norte - Sur en el debate ambiental ha continuado en el tiempo. Un aspecto central para entender este fracaso tuvo que ver con la administración de los fondos a recolectar por parte del denominado Fideicomiso Internacional (FI).

$\mathrm{Al}$ respecto, en julio de 2008, el gobierno del Ecuador constituyó el Consejo Administrativo y Directivo de la Iniciativa Yasuní-ITT(El Consejo) encargado de delinear las políticas para sacar adelante esta iniciativa, trabajar en los mecanismos financieros y desarrollar estrategias de promoción y negociación (Gobierno del Ecuador, 2008 y 2010). Este Consejo estableció negociaciones con el PNUD, para concretar la firma de un FI, que debía ser presentado para la Cumbre sobre Cambio Climático de Copenhague en diciembre de 2009 sin embargo, las negociaciones fueron suspendidas previo a la celebración de esta Cumbre por el Presidente del Ecuador.

La molestia del Ecuador apuntaban a que el PNUD exigía que el país renunciara a su soberanía al plantear que el FI fuera administrado por una mayoría de miembros representantes de los países contribuyentes, lo cual dejaba su administración estratégica -el uso de sus fondos y qué tipos de proyectos llevar adelante con esos mismos fondos-, fuera de la soberanía del sistema público ecuatoriano. En palabras de Correa, el acuerdo pretendido implicaba dejar la dirección de la iniciativa en manos de "una burocracia internacional", por lo cual este les decía: "Saben qué, señores, vayan a mandar a su casa, cambien su donaciones en centavitos y pónganselo en la oreja, porque nosotros no vamos a recibir órdenes de nadie; esa plata es del pueblo ecuatoriano" (Correa, 9 de enero de 2010). A raíz de esta primera crisis se producen cambios en los miembros del Consejo, se convocó a un nuevo equipo que elaboró un nuevo documento de FI, que es aprobado y firmado en agosto de 2010. 
Este nuevo FI seguía siendo administrado por el PNUD, pero el procedimiento para la selección de los proyectos a ejecutar con los dineros del Fondo, incluía un proceso de autorización a cargo de la denominada Entidad de Coordinación Gubernamental, que era un órgano compuesto por el Ministerio de Coordinación del Patrimonio, con lo cual el Ecuador volvía a ejercer soberanía sobre la administración de esta iniciativa (Gobierno del Ecuador y PNUD, 2010).

Por cierto, esta actitud del Presidente ecuatoriano fue criticada por los gobiernos de los Estados Unidos, Gran Bretańa, Alemania y otros países del Primer Mundo, acusándolo de hiper-nacionalista y/o nacionalista económico, y de no dar garantías suficientes a los contribuyentes internacionales, entre otras razones. Posteriormente, se tuvo conocimiento por las filtraciones del caso Wikileaks, que los EE.UU. estaban preocupados por lo que denominaban la "obsesión por la soberanía" que presentaba Correa al insistir en la defensa de los recurso naturales del Ecuador, así como las dificultades que ponía el gobierno ecuatoriano para establecer la cooperación militar con los EE.UU., a lo que se sumó la expulsión de dos agentes norteamericanos en febrero de 2009. Por su parte, el gobierno alemán, que en sus inicios había manifestado gran apoyo retórico a esta iniciativa del Ecuador, en septiembre de 2010 cambió de actitud y la comenzó a boicotear molesto por el rechazo del presidente Correa a dejar el control de la misma en manos internacionales, y en junio de 2011 declaró que su país no aportaría a esta iniciativa. Es importante destacar que el gobierno alemán veía con enorme preocupación la proyección estratégica que significaba la iniciativa ambientalista del Ecuador ya que amenazaba con entregar la ofensiva internacional ambiental a los países de la periferia. Concretamente, el Ministro de Cooperación alemán Dirk Niebel en una carta dirigida a la diputada Verde Ute Koczi, informaba que Alemania no aportaría a esta iniciativa, argumentando que no existían garantías por parte del Ecuador de mantener el petróleo bajo tierra, pero además seńalaba que "la propuesta ecuatoriana podría formar un precedente peligroso para que otros países productores de petróleo realicen pedidos de compensación similares" (Salazar, 2015, pp. 61-63). De esta forma, el gobierno alemán comprometió un aporte de 45,8 millones de dólares, pero al Programa Especial de Reserva de Biosfera Yasuní controlado por la ONU y no al FI que era la propuesta ecuatoriana (Ibíd).

Y si bien Ecuador logró un acuerdo de FI con el PNUD, según sus intereses, la tensión generada con los principales países contribuyentes del Norte finalmente hizo fracasar la iniciativa.

Como era lógico, la propuesta del Ecuador señalaba que si no se reunían los dineros provenientes de la comunidad internacional en los plazos establecidos, Ecuador llevaría a cabo sus planes extractivos en la zona. Esta fue la situación que finalmente se produjo y que llevó, el 15 de agosto de 2013, al gobierno de Rafael Correa a dar por terminada la iniciativa. 
Para el presidente ecuatoriano, la comunidad internacional interpelada (específicamente los países del Norte industrializado y desarrollado), no había respondido adecuadamente, ya que tras seis años de iniciativa solo se habían reunido 13,3 millones de dólares en el Fondo Yasuní, o sea un 0,37\% de lo esperado, además de otros compromisos no directamente vinculados por otros 116 millones de dólares (Correa, 2013). Por lo tanto, los seis años en que la propuesta estuvo operativa, sin que se lograsen reunir los aportes esperados, habían significado una demora a la solución de problemas de "millones de ecuatorianos sin agua potable, niños muriendo o mal creciendo por causas perfectamente evitables, centenas de miles de jóvenes sin infraestructura educativa adecuada, personas sin acceso a la salud" (Ibíd). Sin embargo, el país necesitaba de esos recursos provenientes de la extracción de petróleo para salir de sus condiciones de subdesarrollo señalando que "necesitamos nuestros recursos naturales para superar lo más rápidamente la pobreza, y para un desarrollo soberano. El que les diga lo contrario les está mintiendo" (Ibíd).

De esta forma, si bien Correa pasaba a transformase en el blanco predilecto del ecologismo ecuatoriano e internacional, al pasar de Presidente respetuoso de los derechos de la Madre Naturaleza a político extractivista y "sin conciencia ambiental" (Vásquez, 2015), lo cierto es que solo estaba manifestando la histórica postura del Sur en general y de América Latina en particular, en el debate ambiental global. O sea, protección de la naturaleza y biodiversidad pero sin sacrificar sus opciones al desarrollo y, sobre todo, con el pago de la deuda ecológica que el Norte tiene con el Sur.

\section{CONSIDERACIONES FINALES}

El fracaso de la iniciativa Yasuní-ITT, volvió a sacar a la luz las contradicciones de poder Norte-Sur que siempre han determinado el debate ambiental en el Sistema Internacional. Tempranamente, en la Conferencia de Founex de 1971, el Sur señaló que la crisis ambiental fue generada por el Norte industrializado, o sea por los países centrales o Primer Mundo, quienes alcanzaron el estatus de sociedades hegemónicas y con el más alto estándar de vida gracias a su estilo de desarrollo que generó la llamada "Civilización Industrial" y en este proceso pusieron en jaque al ecosistema planetario. Además, su desarrollo se estructuró y solo fue posible sobre la base de mantener en condiciones de subdesarrollo al Sur.

Desde Founex en adelante, para el Sur el tema ambiental se ha entendido como medio ambiente y desarrollo. Y, tras cuarenta años de debate internacional, cuando supuestamente se habían logrado importantes avances en esta materia, nuevamente reaparecían con fuerza importantes contradicciones, precisamente 
cuando se trataba de propuestas que venían del Sur. O sea, cuando desde el Sur surgían iniciativas novedosas y justas, que recogían parte de sus demandas históricas en este tema, como era la idea de que el Norte pague al Sur por los servicios ambientales que se le prestan, así como desarrollaba aún más sus argumentos proponiendo que el Norte le pague o compense al Sur por la contaminación neta evitada, el Norte las desechaba completamente. Pero además, el Norte no solo consiguió bloquear esta iniciativa, sino que también consiguió dejar ante la opinión pública internacional, particularmente entre el movimiento ecologista, como gran culpable de este fracaso al gobierno "desarrollista" del Ecuador.

Sin embargo, el verdadero problema era y sigue siendo una cuestión de poder. En el caso Yasuní se expresó en los esfuerzos del Norte por sacar la dirección y gestión estratégica de esta iniciativa del Estado Nación Ecuador, lo que significa en otras palabras, y más allá de la retórica ecologista y ambientalista empleada, poner bajo control político internacional y del Norte territorios que hoy día pertenecen al Ecuador.

Como bien se percibe en la lógica del gobierno alemán para hacer fracasar esta iniciativa, la geopolítica del Norte hegemónico no puede dejar de preguntarse ¿qué sucedería si el Primer Mundo acepta la lógica explícita de la propuesta ecuatoriana, de pagar por no extraer el preciado petróleo? Esta propuesta, que evidentemente es de claro contenido ambientalista y ecologista, ¿acaso no sería replicada por otros países periféricos que también dependen de la exportación de commodities, recursos naturales y materias primas al centro? ¿Qué pasaría en el orden internacional económico y geopolítico, si países exportadores de gas, uranio, cobre $\mathrm{u}$ otro recurso natural solicitan al Primer Mundo que se les pague - un precio justo o de acuerdo a lo que dejarían de ganar-, por no explotar estos recursos, a fin de mitigar las externalidades ambientales negativas que estas actividades conllevan? ¿Qué pasaría con el macro sistema económico-industrial y financiero del centro que necesita de manera creciente estos recursos y al más bajo precio posible, tanto para seguir funcionando, como para seguir beneficiándose de las relaciones de intercambio económico internacional?

Son estas interrogantes las que se deben tener en consideración para entender que para el Norte resultaba fundamental que esta iniciativa no prosperara. En este sentido, el destino fracasado de la propuesta Yasuní-ITT, no se debió a los afanes "desarrollistas" del Ecuador. Por el contrario, esta propuesta avanzó demasiado en un espíritu ecologista y conservacionista, al punto que generó, ingenuamente, un abierto desafío a la lógica de poder que predomina en el debate ambiental internacional y que ha explicado las tensiones Norte-Sur en esta tema desde que se instaló en la agenda política internacional. 


\section{REFERENCIAS}

Bifani, Paolo. Medio Ambiente y Desarrollo Sostenible. Madrid: Instituto de Estudios Políticos para América Latina, 1999.

Correa, Rafael. "Discurso del Presidente en la 62 Asamblea General de las Naciones Unidas”. New York, 25 de septiembre de 2007. Disponible en: http://www. presidencia.gob.ec/discursos/ [Consultado el 04 de enero de 2016].

Correa, Rafael (declaración en prensa); "Ecuador pedirá compensación por no explotar su petróleo". El Mercurio, Santiago, 11 de diciembre de 2008, p A5.

Correa, Rafael. Enlace Ciudadano, 9 de enero de 2010. Disponible en: http:// enlaceciudadano.gob.ec/enlaceciudadano154/ [Consultado el 05 de enero de 2016].

Correa, Rafael; Ecuador: de Banana Republic a la No República. Buenos Aires: Debate, 2010.

Correa, Rafael, (Discurso); "XVI Conferencia de las Partes de la Convención Marco de las Naciones Unidas sobre Cambio Climático". Cancún, 8 de Diciembre de 2010. Disponible en: http://www.presidencia.gob.ec/wpcontent/uploads/downloads/2012/10/081210_XVI-Conferencia-de-laspartes-de-la-Convencion-Marco-de-la-Naciones-Unidas-sobre-CambioClimatico.pdf [Consultado el 04 de enero de 2016].

Correa, Rafael, (Discurso); "Primera Cumbre Internacional de Medio Ambiente". Guayaquil, 14 de Marzo de 2011 (a). Disponible en http://www.presidencia.gob. ec/wp-content/uploads/downloads/2012/10/2011-03-14-Primera-CumbreInternacional-de-Medio-Ambiente.pdf [Consultado el 06 de enero de 2016).

Correa, Rafael, "Ponencia del Presidente Rafael Correa en la New School of Social Research Nueva York"; New York, 23 de septiembre de 2011 b. Disponible en: http://www.presidencia.gob.ec/wp-content/uploads/ downloads/2012/10/2011-09-23-Discurso-en-New-School-of-SocialResearch-New-York.pdf [Consultado el 05 de enero de 2016].

Correa, Rafael; Informe a la Nación (Primera a Parte). Quito, 10 de Agosto de 2012. Disponible en: http:/www.presidencia.gob.ec/wp-content/uploads/ downloads/2012/10/2012-08-10-Informe-a-la-Nacion-2012-Primera-Parte. pdf [Consultado el 05 de enero de 2016]. 
Correa, Rafael, (Discurso) "La Iniciativa Yasuní-ITT: cambiando paradigmas para un futuro sustentable”; Río de Janeiro, 20 de junio de 2012. Disponible en: http:// www.presidencia.gob.ec/wp-content/uploads/downloads/2012/10/2012-06-20Iniciativa-Yasuní-ITT-Cambiando-paradigmas-para-un-futuro-sustentable.pdf [Consultado el 05 de enero de 2016].

Correa, Rafael, “Anuncio a la Nación Iniciativa Yasuní-ITT”. Quito, 15 de agosto de 2013. Disponible en: http:/www.presidencia.gob.ec/wp-content/uploads/ downloads/2013/08/2013-08-15-AnuncioYasuní.pdf [Consultado el 04 de enero de 2016].

Estenssoro, Fernando. Medio Ambiente e Ideología. La Discusión pública en Chile, 1992-2002. Antecedentes para una historia de las ideas politicas a inicios del siglo XXI. Santiago de Chile: USACH - Ariadna, 2009.

Estenssoro Saavedra, Fernando. Historia del Debate Ambiental en la Politica Mundial. 1945-1992. La perspectiva latinoamericana. Santiago de Chile: IDEA, 2014.

Gobierno del Ecuador. "Ecuador Yasuní-ITT. Fondo de Fideicomiso: Términos de Referencia”. 28 de julio de 2010. Disponible en: file:///home/jp/ Downloads/Ecuador\%20Yasuní\%20ITT\%20Fund\%20TOR.Spanish\% 203\%20Aug\%202010.pdf [Consultado el 04 de enero de 2016).

Gobierno del Ecuador y Programa de las Naciones Unidas para el Desarrollo. "Memorando de acuerdo para la administración y otros servicios de soporte relacionados con el fondo Ecuador Yasuní- ITT", 3 de agosto de 2010. Disponible en: file:///home/jp/Downloads/Ecuador\%20Yasuní\%20 ITT\%20Fund\%20MOA.Spanish\%203\%20August\%202010.pdf [Consultado el 04 de enero de 2016].

Gobierno del Ecuador. "Plan Nacional para el Buen Vivir 2013-2017”; Secretaría Nacional de Planificación, 2013. Disponible en: http://www.buenvivir. gob.ec/web/guest [Consultado el 05 de enero de 2016].

Grinberg, Michel. Ecofalacias. De cómo las multinacionales se apoderan del discurso del ambiente. Buenos Aires: Galerna, 1999.

Guimaraes, Roberto P.; "El discreto encanto de la cumbre de la tierra. Evaluación impresionista de Río 92”, Nueva Sociedad, No122, (1992): 86-103. 
IPCC; Cambio climático 2007: Informe de sintesis. Contribución de los Grupos de trabajo I, II y III al Cuarto Informe de evaluación del Grupo Intergubernamental de Expertos sobre el Cambio Climático. Ginebra-Suiza: IPCC, 2008. Disponible en: www. un.org/es/globalissues/climatechange [Consultado el 03 de diciembre de 2015].

Larrea, Carlos. "La Iniciativa Yasuní ITT: Una opción falible hacia la equidad y la sustenatbilidad”. En Vallejo, María Cristina et al. La Iniciativa YasuniITTdesde una perspectiva Multicriterial, Quito, 2011.

Larrea, Carlos. "Yasuní ITT. An initiative to change the history”. Quito, Gobierno del Ecuador, 2012 (a). Disponible en: www. Yasuní-itt.gob.com [Consultado el 15 de julio de 2013].

Larrea, Carlos. "Ecuador's Yasuní-ITTInitiative: an options towars equity and sustainability", The Road to Rio + 20, Issue 3, Naciones Unidas, 2012 (b): 58 -63.

Meadows, Dennis L., Meadows, Donella H., Randers, J†rgen, Behrens III, William W. The Limits to Growth. New York: A Potomac Associates Book, 1972.

Murga Menoyo, M. de los Ángeles (coord.). Desarrollo local y Agenda 21. Madrid: Pearson, Prentice Hall, 2006.

Ozorio de Almeida, Miguel A. "El Mito del Equilibrio Ecológico”. El Correo, enero 1973 (año XXVI), pp. 25-28.

Salazar, Fanny. Análisis de la "Diplomacia Verde" del Gobierno Ecuatoriano: caso Yasuni-ITT, enero 2007 a julio 2013. Disertación de grado previa a la obtención del título de Licenciada Multilingüe En Negocios Y Relaciones Internacionales. Pontificia Universidad Católica del Ecuador. Quito, 2015.

Strong, Maurice, "El décimo aniversario de la Conferencia de Estocolmo". En Echechuri, Héctor et.al. Diez Años Después de Estocolmo. Desarrollo, Medio Ambiente y Supervivencia. Madrid: CIFCA, 1983.

Sunkel, Osvaldo. "El capital ecosistémico", Ambiente y Desarrollo, 6 /3 (1990): 23-27.

Sunkel, Osvaldo y Tomassini, Luciano. "Los factores ambientales y el cambio en las relaciones internacionales de los países en desarrollo". En Sunkel O. y Gligo N. (Eds.). Estilos de desarrollo y Medio Ambiente. Ciudad de México: Fondo de Cultura Económica, (1980): 288-319. 
Vásquez, Juan Pablo. Medioambiente, Desarrollo y Soberania: La Tensión Norte sur Global en el Debate Medioambiental. El Caso de la Iniciativa Yasuni-ITTdel Estado Ecuatoriano como Propuesta de Política Pública Global desde América Latina. Tesis para optar al grado de Magíster en Estudios Internacionales, Instituto de Estudios Avanzados, Universidad de Santiago de Chile, 2014.

Vásquez, Juan Pablo. "La tensión histórica Norte-Sur global en el debate ambiental. El conflicto en torno a la iniciativa Yasuní-ITT", Revista Estudios Hemisféricos y Polares, 6/1 (2015):1-28.

\section{Documentos:}

A/RES/2398 (XXIII). Problemas del Medio Humano, 3 de diciembre del 1968. (A/RES/2398 (XXIII), 1968).

República del Ecuador, “Constitución de la República del Ecuador”. Quito: 2008.

"Declaración de la Conferencia de las Naciones Unidas sobre el Medio Humano". En Grinberg, Miguel. Ecofalacias. De cómo las multinacionales se apoderan del discurso del ambiente. Buenos Aires: Galerna, 1999.

"El Informe de Founex"; en Marino de Botero, Margarita y Tokatlian, Juan (comp.). Ecodesarrollo. El pensamiento del decenio. Bogotá: IDERENA/ PNUMA, (1983): 51-85.

\section{Páginas web:}

https://wikileaks.org/plusd/cables/09QUITO937_a.html.

https://wikileaks.org/plusd/cables/09QUITO204_a.html.

https://wikileaks.org/plusd/cables/10QUITO101_a.html. 